\title{
Endoscopic ultrasound guided injection of iron oxide magnetic nano- particles for liver and pancreas: a feasibility study in pigs.
}

\author{
Bogdan Silviu Ungureanu1, Daniel Pirici², Claudiu Mărgăritescu ${ }^{3}$, Ioana Andreea Gheonea ${ }^{4}$, \\ Florian Nicu Trincu ${ }^{5}$, Adrian Fifere ${ }^{6}$, Adrian Săftoiu ${ }^{1,7}$
}

${ }^{1}$ Research Center of Gastroenterology and Hepatology Craiova, University of Medicine and Pharmacy Craiova, Romania, ${ }^{2}$ Department of Research Methodology, University of Medicine and Pharmacy Craiova, Romania, ${ }^{3}$ Department of Pathology, University of Medicine and Pharmacy Craiova, Romania, ${ }^{4}$ Department of Radiology, University of Medicine and Pharmacy Craiova, ${ }^{5}$ Faculty of Pharmacy, University of Medicine and Pharmacy of Craiova, Romania, ${ }^{6}$ Institute of Macromolecular Chemistry “Petru Poni”, Iasi, Romania, ${ }^{7}$ Department of Endoscopy, Gastrointestinal Unit, Copenhagen University Hospital Herlev Copenhagen, Denmark

\begin{abstract}
Aims: Pancreatic cancer and hepatocellular carcinoma are two of the most aggressive types of cancer with limited therapeutic options in stages of advanced disease. Our objective is to assess the safety and feasibility of injecting iron oxide nanoparticles (IONs) via endoscopic ultrasound (EUS)-guidance, both systemically and locally in the liver and pancreas in order to study new potential therapies for liver and pancreatic tumors. Material and methods: Six domestic pigs were used for our study design, and divided into three groups: two were injected in the portal vein, and other four were subjected to local exposure of IONs in the liver and pancreas, two each. The pigs were on a 7 days follow-up and necropsy was performed with their organs harvested. A 3T MRI scan was also performed. Results: All animals underwent an endoscopic ultrasound fine needle injection (EUS-FNI) procedure without any complications. EUS-FNI procedure had an average time of 5 minutes and 21 seconds and consisted of $2 \mathrm{ml}$ of ION injection. No perforations and no risk of potential bleeding were recorded. Macroscopic changes were observed only after pancreatic EUS-FNI. A significant amount of IONs was observed in the liver after local injection and after vascular EUS-FNI. The imaging results were confirmed by pathological examination with most of the IONs accumulated in Ito-like cells, Kupfer cells, and sinusoids. Conclusions: IONs have been widely studied for both diagnostic and therapeutic purposes. Their injection through EUS-guidance may develop new diagnosis strategies as well as curative or palliative therapies in pancreatic and liver tumors.

Keywords: endoscopic ultrasound-fine needle injection, iron oxide nanoparticles, liver, pancreas
\end{abstract}

\section{Introduction}

Pancreatic cancer (PAC) and hepatocellular carcinoma (HCC) are two of the most aggressive malignancies with a grim prognosis, ranking on the $3^{\text {rd }}$ and $4^{\text {th }}$ place in terms of mortality worldwide [1]. Because both diseases are frequently diagnosed in advanced stages (either local

Received 02.11.2015 Accepted 10.12.2015

Med Ultrason

2016, Vol. 18, No 2, 157-162

Corresponding author: Bogdan Silviu Ungureanu

Research Center of Gastroenterology and

Hepatology Craiova, University of Medicine

and Pharmacy

661 Mai Bvd,

200638 Craiova, Romania

Phone/fax: +40 251310.287

E-mail: boboungureanu@gmail.com or metastatic), curative options may be no longer available. This has brought forward the need of new potential therapies, which may prolong the patients' life expectancy and improve their conditions [2,3].

Over the years, nanotechnology biomedical applications have opened a window in the research of new diagnostic and therapeutic settings for HCC and PAC [4,5]. Among the different nanoparticles available, iron oxide nanoparticles (IONs) have received extensive attention due to their availability and properties. From diagnostic procedures in enhanced magnetic resonance imaging (MRI) to local therapies such as magnetic hyperthermia or even as vectors for shipping cytotoxic drugs, IONs have proven to have a good safety profile [6]. A key feature for their biocompatibility and biodistribution is related to their general characteristics of physiochemical design and coating properties [7]. 
Regardless of their purpose and method of distribution, either oral, local, or intravenously, the main objective is to deliver a number of IONs as large as possible in the targeted tissue. Several organs, along with the pancreas and liver can become deposit spaces as the iron may be encapsulated within their cells.

Endoscopic ultrasound (EUS) has evolved since its beginning to a routinely used procedure, which further blends interventional radiology techniques with minimal invasive surgery options. Real time imaging of the pancreas and liver provides important details about the tumor's characteristics and may also serve as a precise setting for guiding therapeutic techniques [8]. After introducing EUS-guided fine needle aspiration, more procedures have surfaced expanding the therapeutic armamentarium of endoscopic therapies $[9,10]$. EUS-guided interventions pose a great potential with a prospect of successful treatment results as compared to current available techniques.

Development and improvements in cancer research have indicated EUS-guided fine needle injection (EUSFNI) as a novel technique for local delivery of specific drugs [11]. The aims of the present study are to assess the safety and feasibility of injecting IONs via EUSguidance, both systemically and locally in the liver and pancreas in order to study new therapies for liver and pancreatic tumors.

\section{Materials and methods}

The procedures were performed according to the European Legislations on animal rights, after obtaining a written approval from the Ethics Committee of the University of Medicine and Pharmacy of Craiova (UMFCV).

IONs were synthesized at the Institute of Macromolecular Chemistry "Petru Poni", Iasi, through the co-precipitation method with citric acid solution. At first, $0.60 \mathrm{~g}$ of Fe$\mathrm{Cl} 3 \times 6 \mathrm{H} 2 \mathrm{O}$ was added to $2 \mathrm{ml}$ deionized water and another solution was prepared by adding $0.21 \mathrm{~g}$ of $\mathrm{FeCl} 24 \mathrm{H} 20$ to $0.5 \mathrm{ml}$ of $2 \mathrm{~m}$ solution of $\mathrm{HCl}$. These substances were vigorously stirred after being added to $10 \mathrm{~mL}$ of DI water with citric acid. The resulting solution was titrated with $2 \mathrm{ml}$ of $5 \mathrm{M}$ of sodium hydroxide and stirred for 30 minutes until a black precipitate was formed, resulting in the $\mathrm{Fe} 3 \mathrm{O} 4$ nanoparticle suspension. The ferrrofluid was heated to $80^{\circ} \mathrm{C}$, left for 2 hours and then centrifuged for 5 minutes at $900 \mathrm{xg}$. As a final step the supernatant was added into water, a process which was repeated several times. Before being injected, the magnetic nanoparticle (MNP) solution was sonicated for a proper dispersion for several minutes.

Six domestic pigs were used for our study design and kept in special conditions. The experimental models were subjected to fasting and liquids for 24 hours, respectively 6 hours before intervention. Premedication was administered intramuscularly and consisted of Ketamine $20 \mathrm{mg} /$ kgc (MSD Animal Health, Germany), Xylazine 2mg/ $\mathrm{kgc}$ (Bioveta A.S., Czech Republic) and Athropine 0.015 $\mathrm{mg} / \mathrm{kgc}$ (Biofarm, Romania). Peripheral access was assured with an $18 \mathrm{G}$ catheter (WellcathPlusTM, Wellmed, Noida, India) positioned on the marginal vein of the ear. The pigs were intubated, maintained under general anesthesia with Propofol 0,5 mg/kgc (Fresenius Kabi Austria GMBH - Austria) continuously, Fentanyl $3 \mu \mathrm{g} / \mathrm{kgc}$ (Actavis Nordis A/S - Denmark) and Pavulone $0,1 \mathrm{mg} / \mathrm{kgc}$ (Pancuronium Bromide, Schering-Plough - USA) while all vital signs were monitored.

All procedures were performed with standard equipment intended for animal use only. The pigs were divided into three groups: two were injected in the portal vein, and other four were subjected to local exposure of IONs in the liver and pancreas, two each. EUS-guided injection of IONs was carried out with a linear array EUS scope (GFUCT140-AL5, Olympus, America), with a large interventional channel, coupled with a corresponding Evis Exera System (Olympus, America) and an AlokaProSound 5500 Ultrasound System (Hitachi-Aloka, Tokio, Japan). EUS-FNI through a 19-gauge needle was preferred for local and portal vein injection. The EUS-scope was passed through an overtube placed into the esophagus and advanced to the stomach until a good position of the targeted organs (liver or pancreas) was obtained (fig 1).

A 19-gauge EUS needle (Boston Scientific, USA) was inserted through the biopsy channel, and $2 \mathrm{ml}$ of MNP solution was directly injected either in the portal vein or directly in the liver or pancreas using real time EUS guidance. For the liver, MNP were injected in the left lobe, while for the pancreas the MNP were directed to the head region. Portal vein EUS-FNI consisted of puncturing the vascular wall under real-time

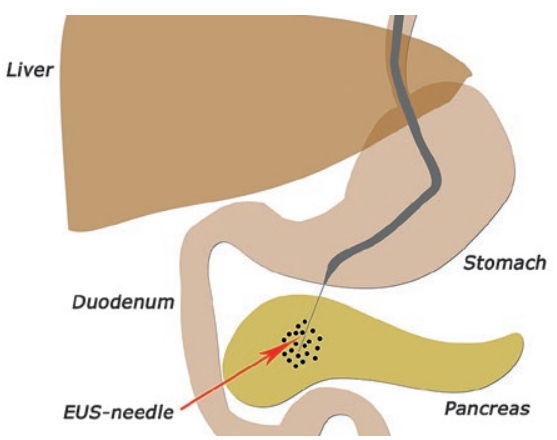

Fig 1. Study design for EUS-FNI in the pancreas on a pig model 


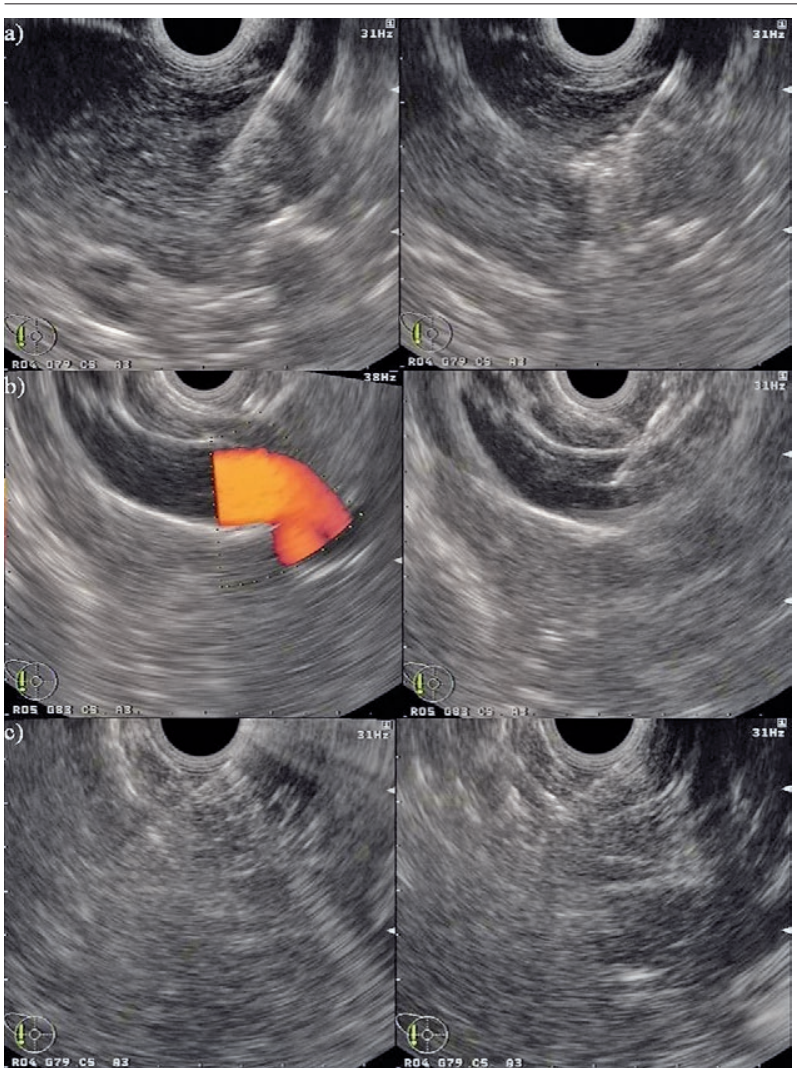

Fig 2. a) EUS-FNI of IONs in the left liver lobe, showing a wide spreading of the ferrofluid solution around the injection area as a hyperechoic mass; b) EUS-FNI in the portal vein pointing out the IONs being dispersed by the bloodstream; c) local EUS-FNI in the head of the pancreas showing hyperechoic images as small quantities of IONs.

EUS guidance and releasing the MNP solution into the bloodstream (fig 2).

The pigs were followed for the next 7 days, with close monitoring regarding any change in their behavior, food intake and body temperature. Animals were euthanized with a pentobarbital overdose and necropsy was performed with their liver and pancreas and other organs being harvested. Kidneys and spleen were also collected to compare the quantity of additional deposits of MNPs on vascular EUS-FNI to local organ injection. Gross examination was performed and organs were stored in buffered neutral formalin and sent for a 3T MRI (Philips Ingenia 3T, Netherlands) scanning with a special research coil.

After routine processing for paraffin embedding, $4 \mu \mathrm{m}$-thick sections were cut from the tissue blocks and were further utilized for hematoxylin-eosin and Prussian blue staining in order to assess the histopathology and ferrous iron deposition. For Prussian blue staining, the slides were incubated in a $2 \%$ potassium ferricyanide acidic solution for 30 minutes at $37^{\circ} \mathrm{C}$, after which

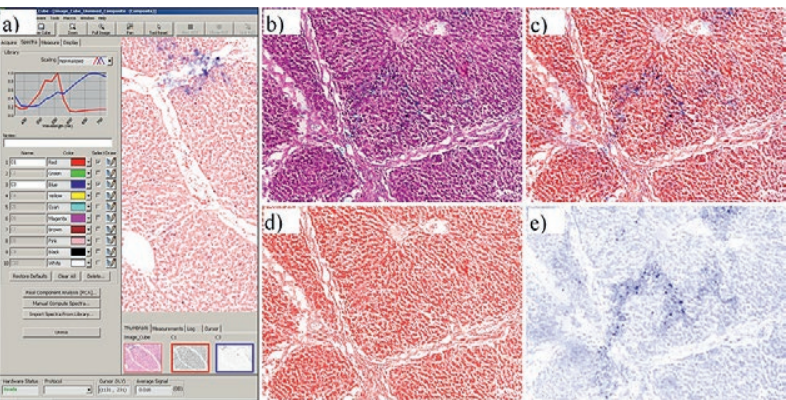

Fig 3. Image processing for spectral unmixing separates signals by unmixing the visible light into $10 \mathrm{~nm}$ bands spectra. Red and blue spectra cubes have been defined (a), and these signatures have been used through the analysis. An exemplary RGB image (b) is shown in pure red and blue signals (c), as well as with individual red (d) and blue channels (e).

they were counterstained with Nuclear Red, dehydrated, cleared and mounted with a xyle-based mounting medium. In order to best separate the Prusian blue and still be able to investigate the morphology of the tissue, the transmission light spectra of the Prussian blue and Nuclear Red were separated by spectral unmixing using a Nuance FX multispectral camera capable of resolving $420-720 \mathrm{~nm}$ spectral range with a resolution of $10 \mathrm{~nm}$, and the Nuance 3.0.2 software (PerkinElmer, Hopkinton MA, USA) (fig 3). Blue signal areas were next calculated on $40 \times$ images centered on either portal spaces or centrolobular veins, averaged (10 image captures for each anatomical region) and means plotted and compared (Microsoft Excel, Microsoft Office 2010).

\section{Results}

All 6 pigs, with a weight range between $25-35 \mathrm{~kg}$ underwent a EUS-FNI procedure without any significant changes in their behavior or any sign of additional complications. No difficulties were encountered in identifying the vascular structures and the targeted organs under EUS-guidance. EUS-FNI procedure had an average time of 5 minutes and 21 seconds. During local EUS-FNI, a hyperechoic mass was created at the injection point, having a median diameter of $1.5 \mathrm{~cm}$ in the liver and $1.2 \mathrm{~cm}$ in the pancreas. After catheterization of the portal vein, the MNP solution was dispersed into the bloodstream with no immediate sign of thrombosis. No perforations of the gastric wall or other organs and no risk of potential bleeding were recorded during the procedures.

Necropsy results showed no signs of local or distant complications. Macroscopic changes were observed only after local injection in the pancreas with a black spot highlighting the concentration point where the MNP were injected (fig 4). 


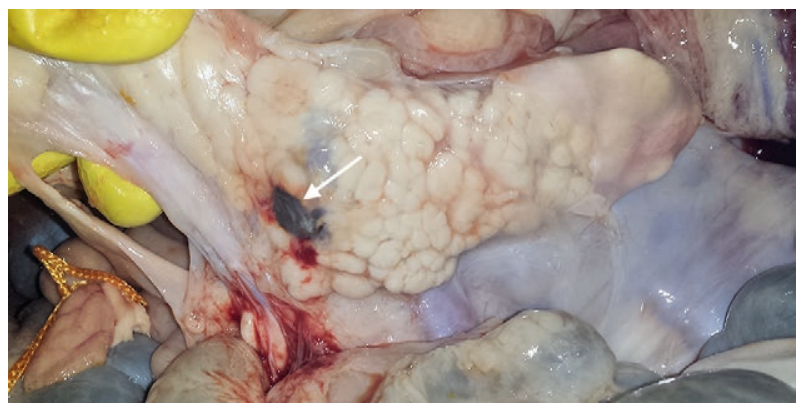

Fig 4. Necropsy reveals a large deposit of IONs as a black spot at the injection point in the head of the pancreas (arrow)

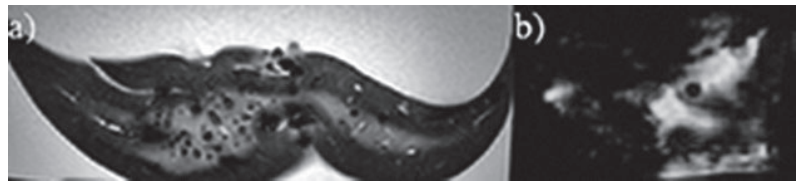

Fig 5. 3T MRI images recorded after organ's harvesting pointing out deposits of IONs within the liver a) and the pancreas b).

The organs were evaluated by 3 Tesla (3T) MRI scanning, which hallmarked MNP concentrations in the liver and pancreas. A significant amount of IONs was observed in the liver after local injection and after vascular EUSFNI. Portal vein injection showed no sign of thrombosis, even though there were deposits of MNPs even in the periphery area (fig 5a). Regarding the pancreas, MRI images were only relevant after local EUS-FNI procedures (fig 5 b).

Pathological assessment showed various deposits within the selected organs. Local injection in the liver showed a large volume of MNPs in the targeted area and several deposits in small quantities further away diffusing into the parenchyma. In contrast, EUS-FNI in the portal vein revealed a large amount of IONs scattered in the hepatic lobules, as well as between the lobules and in the hepatic periportal space.

Initial hematoxylin and eosin staining revealed clearcut iron pigment deposition in the portal veins and some of the peripheral sinusoids (fig 6a), with no visible hemosiderin accumulation in and around the central veins (fig 6 b,c). More than $80 \%$ of all the visualized portal spaces presented this accumulation. However, when we utilized the specific Perl's Prussian Blue histological staining, it became clear that the extent of deposition was much larger, with diffuse depositions in the lobule around the central vein (fig $6 \mathrm{~d}, \mathrm{e}, \mathrm{f}$ ), the density of the deposits increasing gradually with shorter distances to the portal spaces (fig $6 \mathrm{~g}, \mathrm{~h}$ ). The pigment was so dense in the portal vein, that the spectral unmixing visualized the reddish appearance under the blue dense shade of Perl's staining. None of the intralobular deposits showed the same bi-phasic spectral signature due most probably to

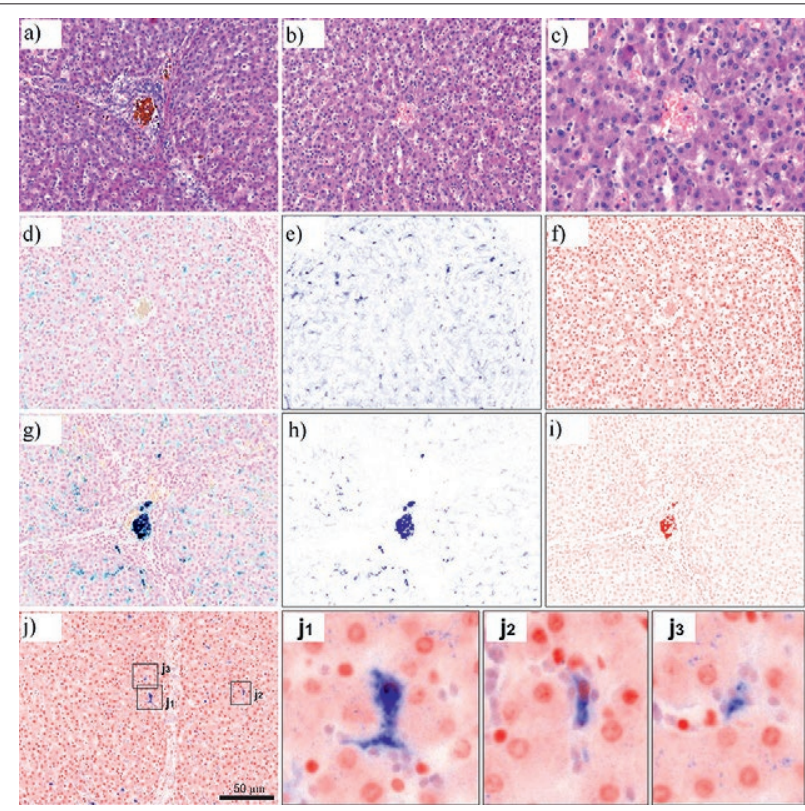

Fig 6. Pathological assessment of the iron deposition in the liver. Only dense hemosiderin pigment is identified on hematoxylin and eosin, mainly in and around the portal spaces (a), while almost no pigment can be identified around the centrolobular spaces (b-c). Spectral unmixing on Perl's stained slides reveals more diffuse depositions around the central lobular areas (d,e,f) compared to peripheral areas ( $\mathrm{g}, \mathrm{h}, \mathrm{i})$. Most of the diffuse depositions appeared to be accumulating in Ito-like cells $(\mathrm{j}, \mathrm{j} 1)$, Kupfer cells $(j, j 2)$ and in the sinusoids $(j, j 3)$.

the dilution of the particles downstream the blood flow. High resolution images revealed that most of the diffuse deposits in the lobule were present in Ito-like cells (fig 6 $\mathrm{j}_{1}$ ), Kupffer cells (fig $6 \mathrm{j}_{2}$ ) or in the sinusoid themselves (fig $6 \mathrm{j}_{3}$ ). Only on rare occasions it seems that the deposits occurred in the hepatocytes.

When we evaluated the blue areas in Perl's staining, this showed that despite the fact that the densest deposits were situated around the portal spaces, these were not the largest. This analysis showed that blue areas around centrolobular spaces were significantly larger $\left(27019.85 \pm 3290.38 \mu \mathrm{m}^{2}\right.$ and $\left.15589.53 \pm 6526.35 \mu \mathrm{m}^{2}\right)$ than those around the periportal areas $\left(3290.38 \pm 195.16 \mu \mathrm{m}^{2}\right.$ and $6526.35 \pm 290.62 \mu \mathrm{m}^{2}$ ), and this staining decreased from perihilar regions compared to peripheric lobar areas. Periportal regions had no such variation between the two anatomical sampling sites, and all instances showed significantly higher values compared to the control corresponding liver areas (fig 7).

Local pancreas EUS-FNI showed a large area of MNP deposits with a localized inflammatory tissue reaction at about $2 \mathrm{~cm}$ away from the injected area. A mild inflammation of the pancreatic parenchyma with fat necrosis and atrophy characterized the surrounding tissues. 


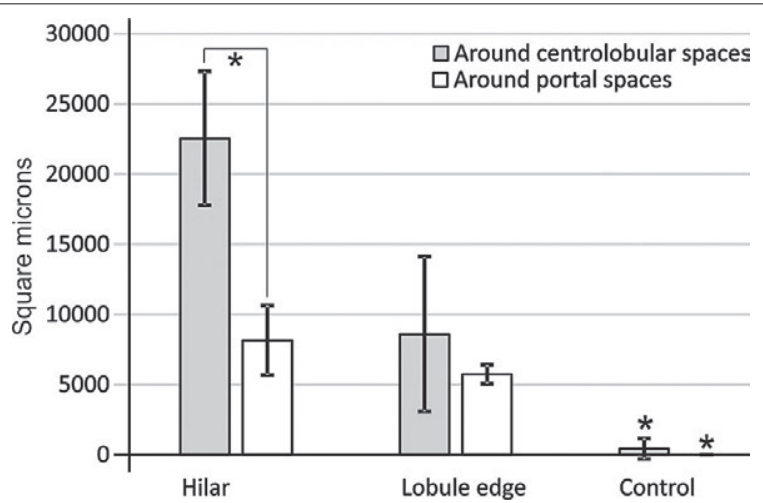

Fig 7. Semiquantitative analysis of Perl's staining shows decreasing staining areas from the hilar to peripheral lobar regions. At the histological level, the areas were much larger around the centrolobular spaces compared to the periportal regions, and the difference was significant only for hilar anatomical regions. All injection sites demonstrated a larger signal area compared to the control tissue. * represents a significance on Student's t testing of $p<0.05$. Bars represent standard error of the means.

\section{Discussions}

Treatment options for patients with liver and pancreatic tumors are known to be relatively limited if the disease is diagnosed in an advanced stage. Lately, magnetic nanoparticles have served as diagnostic tools in contrast imaging MRI in humans [12] and as therapeutic methods such as hyperthermia, drug carriers or gene therapies in experimental models [13]. Tissue reactions to MNP's physico-chemical properties are known to be influenced by their general properties and coatings, biocompatibility and toxicity and nonetheless methods of distribution [14]. Depending on their purpose they have been injected locally within a region of interest, orally after gavage following the absorption route or intravenously trying to direct them to the tumor. However, these techniques are rather difficult to control as the biological response of the targeted tissue requires a large amount of MNPs to fulfill the therapeutic effect [15]. Generally, vascular injection and distribution of MNPs require their passage through three phases: blood stream clearance, extravasation and interstitial space depositing from where they may attack the cancer cells. So far, there are few studies that exploit the potential of nearby vascular structures injection mainly because of their difficult accessibility. EUS may be able to fill up this gap, because of its great imaging potential and various therapeutic options.

Our study focused on the distribution of MNPs through several methods of injection trying to emphasize their liver and pancreas enhancement. EUS-FNI may be taken into consideration as a palliative option either by local injection or by gaining vascular access. Its potential in diagnostic and therapeutic settings based on real-time imaging may overcome the flaws of interventional radiology. There is no doubt that EUS-guidance provides a more attractive option for vascular therapies, as both major and smaller vessels near the gastrointestinal tract can be traced and easily accessed.

EUS-FNI has the ability to target the liver and pancreas using a transgastric and transduodenal approach. So far this setting has been tested with intratumoral ethanol injection [16,17] and chemotherapeutic drugs [18]. EUS-FNI ethanol injection in the pancreas in a porcine model has been proven to be technically possible and rather safe with mild side effects [16]. Our results after local MNP injection in the pancreas showed no evidence of an important pancreatic reaction confirming that EUSguided injection is technically successful because of the nearby position of the pancreas near the gastric wall. Similar results were noted by Kai et al [19] who injected an OncoGel solution in a porcine pancreas tail and observed the deposits 14 days after exposure proving that the technique is feasible and could be a potential therapy for advanced pancreatic cancer.

Liver delivery of MNP was assessed while using different ways of administration. EUS-FNI local delivery showed a large amount of MNP concentrated in the targeted area with only small concentrations in the surrounding liver tissue. Even though there are concise protocols for patients with HCC tumors, the use of EUSFNI has been noted so far in animal settings, as well as some case reports $[20,21]$. On the other hand vascular access in the portal vein and delivery of MNP showed a wider diffusion of MNP in the entire liver, up to the distal branches. This setting seems to be a more appropriate option if a larger number of tumors are targeted.

Local EUS-FNI and portal vein EUS-FNI might be used for different tumor types. While local injection may be addressed to a solitary liver tumor (such as HCC or solitary metastases focusing on a larger concentration of MNP in the region of interest), the potential of portal vein injection could be directed in hepatic metastases covering most of the liver tissue. Also, specific coated MNP with different chemotherapeutic drugs injected through the portal vein could lower the systemic toxic effects caused by peripheral injection. This setting was studied by Faigel et al [22] who compared the level of several chemotherapeutic drugs after injecting them in the portal vein and systemically in several experimental pig models. After comparing the results he observed that irinotecan, doxorubicin loaded microbeads and paclitaxel nanoparticles had higher concentrations in the liver tissue after a portal vein injection as compared to a systemic injection, while toxic levels were almost halved especially in the cardiac tissue. MRI images were consistent 
with the pathological examination when comparing local EUS-FNI to portal vein access.

This study has several limitations consisting of the small number of animals and the fact that the MNP distribution was assessed only with MRI scanning and pathological examination. While trying to validate the EUS technique we did not measure the iron concentration within the tissues and also did not focus on urinary samples to see the MNP elimination. Nevertheless, the MNP concentration can be easily estimated through imaging, leading to a better quantification inside the desired region of interest that will be targeted through magnetic hyperthermia.

\section{Conclusions}

Our study focused on showing the feasibility of EUSFNI of MNPs in the liver and pancreas by local or vascular access and their organ distribution. EUS-guidance offers a unique access to these organs and nonetheless to nearby vascular structures, which facilitates desirable therapeutic techniques, which have been the target of interventional radiology over the years. MNPs have been widely studied for HCC and PAC in different therapeutic scenarios such as hyperthermia or coated with different chemotherapeutic drugs. Their injection through EUS-guidance may develop new strategies of diagnosis or curative or palliative therapies in pancreatic and liver tumors.

Acknowledgement: This article was financed by the Partnership program in priority areas - PN II, implemented with support from National Authority of Scientific Research (ANCS), CNDI - UEFISCDI, project nr. 2011-3.1-0252 (NANO-ABLATION).

\section{Conflict of interest: none}

\section{References}

1. Ferlay J, Shin HR, Bray F, et al. GLOBOCAN 2012. Accessed on 10/10/2015.

2. Galun D, Basaric D, Zuvela M, et al. Hepatocellular carcinoma: from clinical practice to evidence-based treatment protocols. World J Hepatol 2015; 7: 2274-2291.

3. Cid-Arregui A, Juarez V. Perspectives in the treatment of pancreatic adenocarcinoma. World J Gastroenterol 2015; 21: 9297-9316.

4. Rosenberger I, Strauss A, Dobiasch S, et al. Targeted diagnostic magnetic nanoparticles for medical imaging of pancreatic cancer. J Control Release 2015; 214: 76-84.

5. Yan SY, Chen MM, Fan JG, et al. Therapeutic mechanism of treating SMMC-7721 liver cancer cells with magnetic fluid hyperthermia using $\mathrm{Fe}_{2} \mathrm{O}_{3}$ nanoparticles. Braz J Med Biol Res 2014; 47: 947-959.
6. Krishnan KM. Biomedical Nanomagnetics: A spin through possibilities in imaging, diagnostics and therapy. IEEE Trans Magn 2010; 46: 2523-2558.

7. Gobbo OL, Sjaastad K, Radomski MW, Volkov Y, PrinaMello A. Magnetic nanoparticles in cancer theranostics. Theranostics 2015; 5: 1249-1263.

8. Săftoiu A. State-of-the-art imaging techniques in endoscopic ultrasound. World J Gastroenterol 2011; 17: 691-696.

9. Vilmann P, Jacobsen GK, Henriksen FW, Hancke S. Endoscopic ultrasonography with guided fine needle aspiration biopsy in pancreatic disease. Gastrointest Endosc 1992; 38: 172-173.

10. Costache MI, Iordache S, Karstensen JG, Săftoiu A, Vilmann P. Endoscopic ultrasound-guided fine needle aspiration: from the past to the future. Endosc Ultrasound 2013; 2: 77-85.

11. Yan BM, Van Dam J. Endoscopic ultrasound-guided intratumoural therapy for pancreatic cancer. Can J Gastroenterol 2008; 22: 405-410.

12. Li L, Jiang W, Luo K, et al. Superparamagnetic iron oxide nanoparticles as MRI contrast agents for non-invasive stem cell labeling and tracking. Theranostics 2013; 3: 595-615.

13. Yu Y, Sun D. Super paramagnetic iron oxide nanoparticle "theranostics" for multimodality tumor imaging, gene delivery, targeted drug and prodrug delivery. Expert Rev Clin Pharmacol 2010; 3: 117-130.

14. Trincu NF, Balseanu TA, Ungureanu BS, et al. Blood clearance of citric acid-coated superparamagnetic iron oxide nanoparticles in rats - a pilot study. Current Health Sciences Journal 2015; 41: 303-306.

15. Antonelli A, Magnani M. Red blood cells as carriers of iron oxide-based contrast agents for diagnostic applications. J Biomed Nanotechnol 2014; 10: 1732-1750.

16. Zhang WY, Li ZS, Jin ZD. Endoscopic ultrasound-guided ethanol ablation therapy for tumors. World J Gastroenterol 2013; 19: 3397-3403.

17. Park do H, Choi JH, Oh D, et al. Endoscopic ultrasonography-guided ethanol ablation for small pancreatic neuroendocrine tumors: results of a pilot study. Clin Endosc 2015; 48: $158-164$.

18. Sharma V, Rana SS, Bhasin DK. Endoscopic ultrasound guided interventional procedures. World J Gastrointest Endosc 2015; 7: 628-642.

19. Matthes K, Mino-Kenudson M, Sahani DV, et al. EUS guided injection of paclitaxel (OncoGel) provides therapeutic drug concentrations in the porcine pancreas (with video). Gastrointest Endosc 2007; 65: 448-453.

20. Klapman JB, Chang KJ. Endoscopic ultrasound-guided fine-needle injection. Gastrointest Endosc Clin N Am 2005; 15: 169-177.

21. Verna EC, Dhar V. Endoscopic ultrasound-guided fine needle injection for cancer therapy: the evolving role of therapeutic endoscopic ultrasound. Therap Adv Gastroenterol 2008; 1: 103-109.

22. Faigel D, Lake D, Landreth T, Kelman C, Marler R. Endoscopic ultrasonography-guided portal injection chemotherapy for hepatic metastases Gastrointest Endosc 2014; 3(Suppl 1): S1. 